\title{
ON 3D DIMENSION: STUDY CASES FOR ARCHAEOLOGICAL SITES
}

\author{
Maria Grazia D'Urso ${ }^{a}{ }^{*}$, Constantino Luis Marino ${ }^{b}$, A. Rotondi ${ }^{c}$ \\ a DICeM, Department of Civil and Mechanical Engineering, University of Cassino and Southern Lazio, \\ Via G. Di Biasio, 4303043 Cassino (Frosinone), Italy \\ durso@unicas.it \\ ${ }^{\mathrm{b}}$ International Surveyance Company, Via Bianco 9-17 84081 Baronissi (Salerno), Italy \\ cm99@hotmail.it \\ c PhD Student, DICeM, Department of Civil and Mechanical Engineering, University of Cassino and Southern Lazio, \\ Via G. Di Biasio, 4303043 Cassino (Frosinone), Italy \\ a.rotondi@unicas.it
}

\section{COMMISSION VI, Working Group VI/3}

KEY WORDS: camera, 3D, photogrammetric measurement, optics, stereographical, sensor.

\begin{abstract}
:
For more than a century the tridimensional vision has been of interest for scientists and users in several fields of application. The mathematical bases have remained substantially unchanged but only the new technologies have allowed us to make the vision really impressive. Photography opens new frontiers and has enriched of physical, mathematical, chemical, informatical and topographic notions by making the images so real to make the observer fully immersed into the represented scene.

By means of active googless the 3D digital technique, commonly used for video games, makes possible animations without limitations in the dimension of the images thanks to the improved performances of the graphic processor units and related hardware components.

In this paper we illustrate an experience made by the students of the MSc'degree course of Topography, active at the University of Cassino and Southern Lazio, in which the photography has been applied as an innovative technique for the surveying of cultural heritage. The tests foresee the use of traditional techniques of survey with 3D digital images and use of GPS sensors.

The ultimate objective of our experience is the insertion in the web, allowing us the visualization of the 3D images equipped with all data.

In conclusion these new methods of survey allow for the fusion of extremely different techniques, in such an impressive way to make them inseparable and justifying the origin of the neologism "Geomatics" coined at the Laval University (Canada) during the eighties.
\end{abstract}

\section{INTRODUCTION}

In the past the traditional methods of stereoscopy and photogrammetry have been characterized by significant difficulties of vision; they employed complex and huge instrumentations and were used almost exclusively by specialized personnel. In fact stereoscopy required highly qualified users which needed to be accustomed to the stereo vision.

Conversely, the new frontier of 3D photograph effective, so that the advantages for people having past experience are highlighted by the capability to reproduce reality in a unified manner with a full immersion in photography. Moreover 3D stereoscopy frees vision from the use of classic (red-cyan filter) lenses and associated systems.

The increase in the sensors capabilities and the software control via software of the optic aberrations have led to a very high definition with the possibility to identify objects within shadows for several diaphragm values. Besides, in order to enhance resolution, the employment of free filter cameras has required the correction of some collateral aberration phenomena.
During the measurements carried out within the study cases illustrated in the sequel we have tried, as much as possible, to avoid macroscopic errors by means of "ad hoc" technologies. In this respect it's worth being mentioned the well know error committed by the NASA technicians relatively to the first blurry images taken in the space.

In fact, in 1972, in the conclusive report concerning the blurred images taken in the space by the Sonar $56 / 250 \mathrm{~mm}$ with a maximum opening diaphragm of $5.6 \mathrm{~mm}$, the NASA relators, Fritz Determann and Heinz Zajadatz stated that " the Zeiss optics, used in that experience, showed a poor (achromatization) based an absolute optical scheme of the 1932, that is a tele - lens put on the market in the 50's, used with a full opening with a red filter necessary for the lunar shots".

Subsequently, about four years later, the Zeiss company put on the market the 5.6/250 mm Superachromat Sonar, a super - chromatical lens which carried out a by far more correct secondary spectrum thanks to the incorporated fluorite lens. In fact, this lens used low - dispersion glasses in place of the crystalline materials used in the past which were more fragile and sensitive to temperature leaps.

\footnotetext{
* Corresponding author.
} 
This example highlights the importance of knowing the innovative techniques and their use more appropriate to the problem at hand.

To this end, within the activities related to the Topography course, held at the University of Cassino and Southern Lazio, graduate students of Civil and Environmental Engineering took part to a practical experience of photogrammetric shots in the archaeological sites of Pompei and Paestum, in the South of Italy.

They had this experience after having attended two introductive seminars called:" First elements of photography and photogrammetry" and "Photogrammetry and Stereoscopy for the built rehabilitation".

\section{OUTLINE OF STEREOSCOPHIC METHODS AND TECHNIQUES}

It is well known that stereoscopy is based on the idea that two photograms taken at two points whose relative distance is approximatively $6.5 \mathrm{~mm}$, generate two images which blend together to produce an "anaglyph".

This fusion of images reproduces the binocular vision of the human eye, known as "stereopsys", i.e. the natural 3D stereoscopic vision of men.

The superimposition of the two digital images creates a "digital anaglyph" whose format is generally defined mpo, i.e. multi picture object, the standard extended format (CIPA DC-007-2009) developed by Camera \& Imaging Products Association, Japan (2009).

Tests carried out in this experience require the use of traditional techniques of surveying with production of 3D digital images in the mpo format and employment of GPS sensors for geo - referencing. The reading and the measurement of the monuments turned out to be particularly effective by adopting the mpo format and gave the possibility to control cracks and degradation, otherwise not possible with the use of separate technologies.

The adopted cameras, calibrated and corrected via software, ensure a precision whose order of magnitude is of $10^{-2} \mathrm{~mm}$. In these conditions a single measurement session allows one: a volumetric measurement and to set the bases for the structural analysis of the monuments; to monitor and establish the health state of bronzes and stone materials; the monuments geo-referencing with associated automatic introduction in the maps; 3D vision with full immersion in the structure and, last but not least, the introduction of highquality images into web sites like Nikon Image Space and Google maps with immediate diffusion for an unlimited number of fixed users and palmars.

This is represented in Figure 1, where it is illustrated an image can be downloaded in mpo format from the website www.nikonimagespace.com.

The errors which are frequently experienced in photogrammetric measurements are mainly due to two different causes: use of compact amatorial cameras characterized by unknown sensibility values which can automatically increase; lack of stabilizers inside the cameras, with long shooting times that induce "micro - movements" phenomena; digital files which are algorithmically and automatically compressed as function of often poor optics features; use of diaphragms that don't optimize the optics performances.

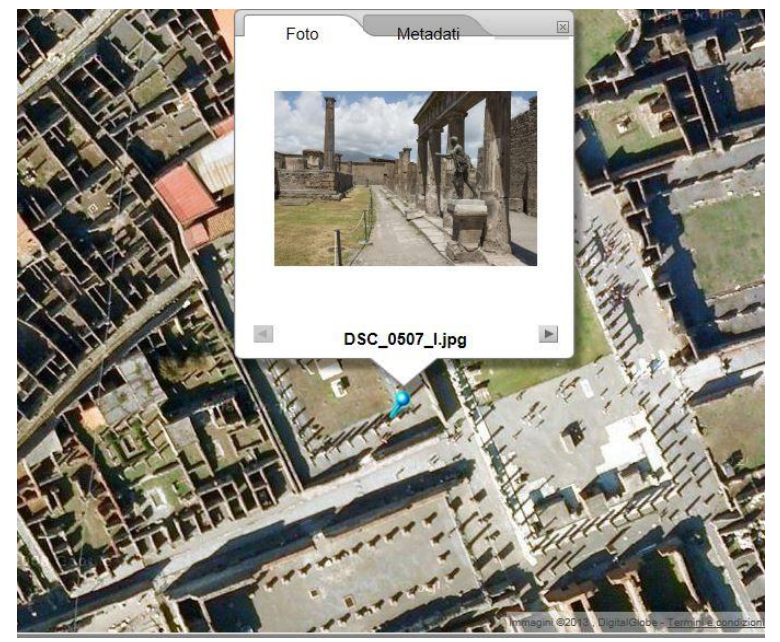

Figure 1. Apollo's Temple image on Nikon Image Space

All these characteristics provide a poor quality of the photograph which is not suited to an ordinary use and exhibit inaccuracies, significant in some cases, making useless the employment of a total station for the baseline measurement.

Conversely, two calibrated cameras have been used in this experience, a H5D - 60 Hasselblad and a Nikon d800 E with a resolution value which is currently among the highest ones in the market. In particular the second camera has been preferred, on one side, for its manageability and light weight, what avoided the use of tripodes, and, on the other one, for the possibility to use low ISO sensibility values and better diaphragms.

The shots taken at intervals and with different lights allow one an improved reading and new possibilities of measurements due to the fact that two different colors can become equal under a specific light. This effects is know as "metameric" phenomenon.

Adopting this procedure the volumes of Apollo's Temple and of the statues, placed into the archaeological site of Pompei; have been determined; moreover, the weight acting on stone structures has been estimated and potential risks ascertained. The new photo resolutions allow the user a close - up vision with scale - values of 10:1. In this way a "raster" mathematic model can be generated.

\section{PHOTOGRAMMETRIC SHOTS OF ARCHAEOLOGICAL SITES}

In both didactic experiences carried out inside the Apollo's Temple, in the archeological site of Pompei, and inside Hera Argiva's Temple, in the archaeological site of Paestum, the postulates of traditional stereoscopy have been strictly followed. In particular, stereoscopic shots have been taken using very high definition calibrated cameras, placed on a micro - metric plate in order to move the shot point exactly of $6.5 \mathrm{~mm}$ along a single axis.

\subsection{Archaeological area of Pompei}

Apollo's Temple is a Roman Temple, buried by the Vesuvio's eruption dated 79 a. D. and discovered after the archeological excavation of ancient Pompei begun in the first half of 1700. It is one of the oldest temples of the ancient city as well as the most visited one for many years. The 
construction of the Temple goes back to the VIII or VII century b. C., as some archaeological findings witness, and it is located in a sacred area where some altars had been erected.

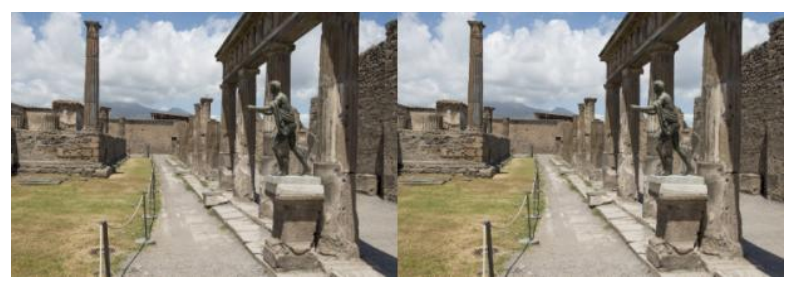

Figure 2. Stereoscopic shots Apollo's Temple right side

In the photogrammetric experience carried out in the Apollo's Temple, see Figure 2, the first stereoscopic shot, i.e. the anaglyph, has been acquired with a Nikon d800E camera from Via Marina which represents the unique access to the temple and is placed on its right side.

Conversely the second stereoscopic shot, taken from the left side of the temple, has been acquired with a Hasselblad H5D60 camera, as it is shown in Figure 3.

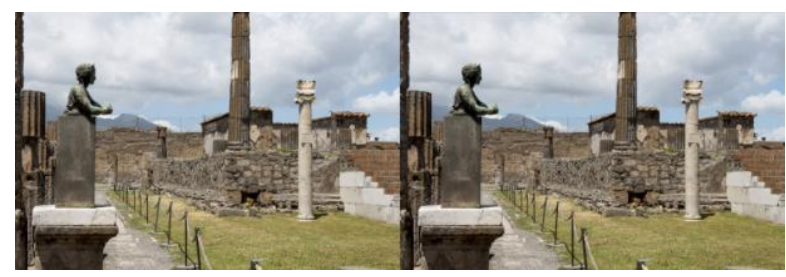

Figure 3. Stereoscopic shots Apollo's Temple left side

The color histogram for the Nikon d800 E camera is shown in Figure 4, referred to the standard value of ISO 100 sensibility, focal length of $35 \mathrm{~mm}$, focus value f13, i.e. focal length/effective diameter optics equal to 13 , and shooting time equal to $1 / 160$ second. These values optimize the acquisition of the images

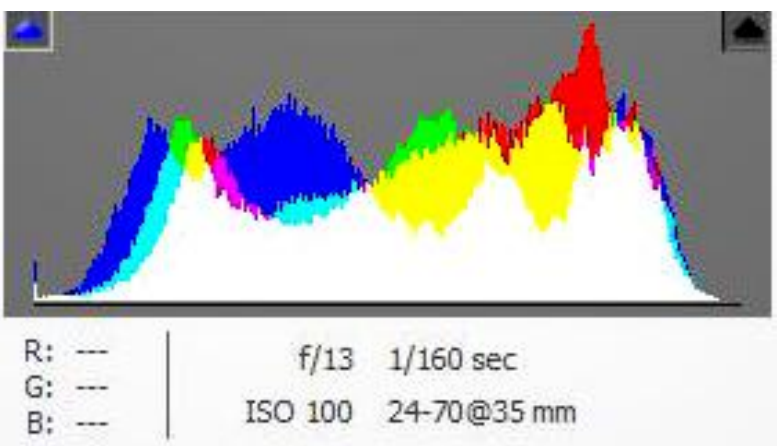

Figure 4. Color histogram camera Nikon d800E

Since low-pass filter-less cameras have been used in both cases, software correction of collateral phenomena has been required, in order to emphasize the resolution

In fact, as it is known, the presence of a shade glass partly reduces the clearness of the image as well as collateral phenomena of optics aberration, e.g. "moire" effect, i.e. the noise caused by the sensors heat that falses the images.
Since the beginning of 1900 the Nobel prize Ostwald (1909) observed that two colors, having different spectrum could not necessarily be distinguished by an observer. In fact two different colors can appear to be similar under a specific light. In order to account for this issue we have taken the shots at different times, with variable lights, so that the shots allowed us to catch details which, otherwise, could have not been evident.

The binary image, in its original digital binary format, has been processed into a digital dark camera, where it is possible to change and correct hundred of parameters. A classic example of correction of the photograms parameters is reported in Figure 5.

The corrections of the photograms parameters are necessary due to the raw format which has been employed, what necessarily requires a post - processing elaboration. In fact the "raw" format is, by definition, a coarse format, in which digital information are not elaborated by the camera, but, a posteriori by "ad hoc" softwares or hardware units.

Thanks to these expedients it is possible to control the balance of the white color, the color temperature, the exposure, the contrast, the lights, the shadows, the clearness, the luminance, the corrections of the lens's aberrations.

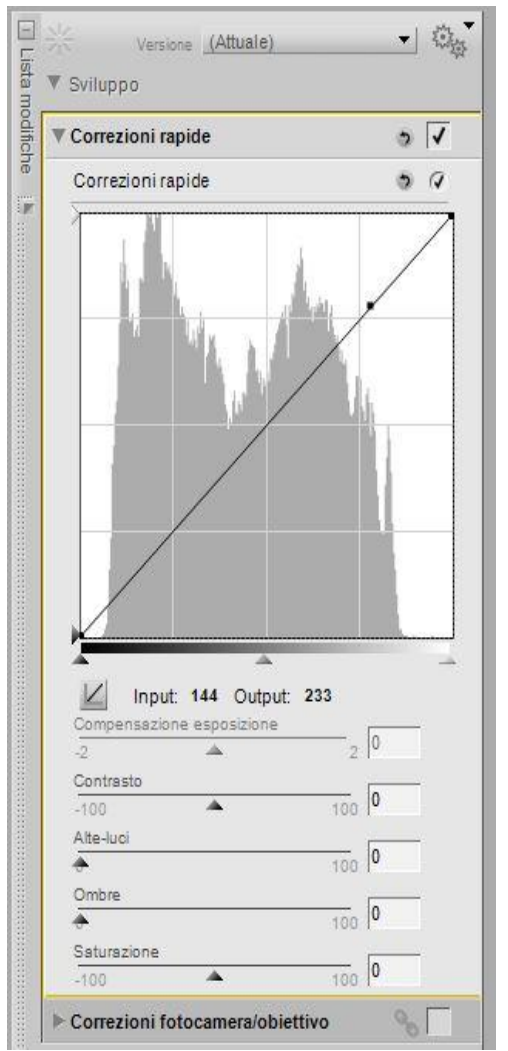

Figure 5. Corrections of camera Nikon

Moreover Figure 6 shows the performance values relative to the Nikon photocamera credited by Metrologic French Laboratory DXO (site: DXO Mark.com France) which certify the exceptionality of the sensor values; at the moment, they are unreached in the market.

Likewise files for the management of the images are now well developed allowing the manipulation of new files; this gives an idea of the enormous amount of data which can be 
obtained from current digital images respect to those available only few years ago.

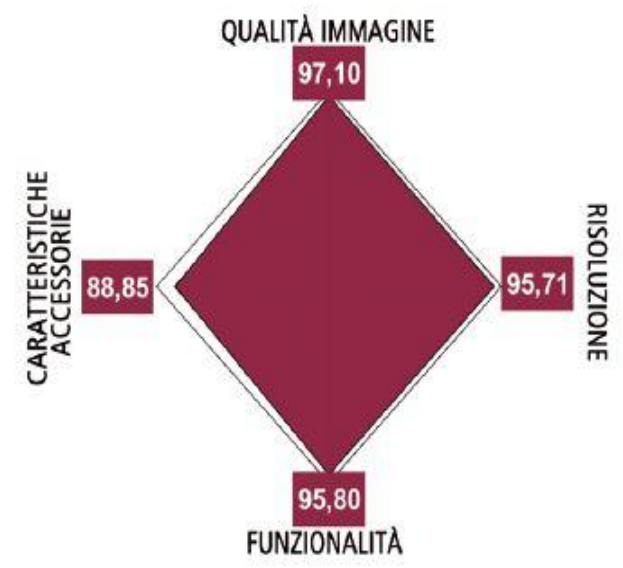

Figure 6. Evaluation test of camera Nikon (Origin: DXO, France)

Actually, it is nowadays available an evolution of softwares able to manage numerical data obtained from digital images. During this experience it has been possible to use these softwares; in particular the dimension of the images shown in the sequel, whose size amount to 300 Megapixel approximately, turns out to be 100 times greater than the dimension of a file obtained by professional cameras produced in 2005.

Moreover the use of active glasses with liquid crystal lenses, similar to those used in videogames, in substitution of the classical stereoscopic instrumentations has allowed the vision of tridimensional images projectable on PC, smart phone, video, etc.

Liquid crystal lenses are a system of glasses with alternate darkening, introduced on the market at beginning new millennium. This system employs electronic 3D glasses, with lenses made of a little liquid crystal display.

The system is governed by a processor and it can be used for the vision of digital images and for the projection of 3D movies. The lenses, synchronized by a processor at the frequency of $100-120$ Hertz of the display, obscurate each eye alternatively; in this way, when on the screen it is visible the image for the left eye, the right lens is obscurated and vice versa.

At such an high frequency, i.e. 100-120 Hertz, two separate images are observed simultaneously and the brain elaborates them by forming an unique $3 \mathrm{D}$ image. In other words the combined effect of the information received from each eye creates a tridimensional effect. This digital anaglyph, in mpo format, has been subsequently compared with a traditional anaglyph.

Figure 7 shows the traditional anaglyph red - cyan relative to Apollo's Temple, visible as a 3D image with the use of standard red -cyan glasses.

As we know from classical stereoscopy a tridimensional image anaglyph is originated, both during projection and printing, from the fusion of two images taken at the distance of human eyes and projected or printed simultaneously: one of the two images is filtered by a red filter and the other one by the complementary colour, which can be blue, green or cyan colour.

It is apparent in Figure 7 that the anaglyph produced as detailed above is the cheapest system to obtain the idea of the depth in standard 2D images, whether they are printed or projected. Accordingly, by using adequate software, it is possible to filter a pair of stereoscopic images, colour or black/white, by producing at the end a digital image in mpo format..

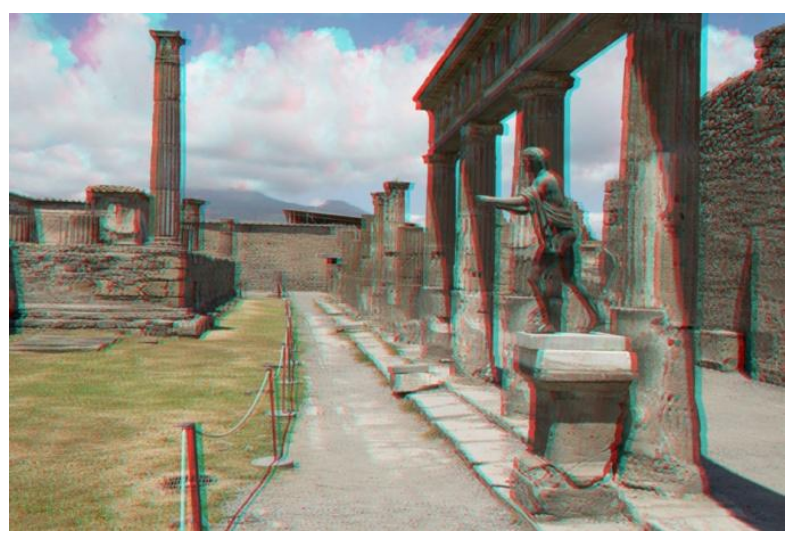

Figure 7. Anaglyph red - cyan of Apollo’s Temple

\subsection{Archaeological area of Paestum}

A further didactic experience made with MSc's graduate students of the course "Topography, GIS and Monitoring Techniques" held at University of Cassino and Southern Lazio, has been carried out inside a very suggestive archeological area in Campania, in the south of Italy. The experience has concerned the high precision photogrammetric surveying of Hera Argiva's Temple, inside the archaeological excavations of Paestum, ex Poseidonia, an old centre of Magna Graecia.

Hera Argiva's Temple, shown in Figure 8, dates back to the half of the $\mathrm{V}$ century b.C. (530 b.C.) when, probably, Poseidonia was a very reach town. The temple, erected in Doric style, has nine columns put along the fronts (peripteral ennea stylus 24,50 meters long) and eighteen columns along the sides $(54,30$ meters); it is characterized by a rate widht/length which fulfills the standards of 2:1 termed "analoghia".

The temple, made of archaic elements which are typical of the classical period, has dimensions lightly smaller, but longer, than Zeus' Temple in Olimpia, from which it is inspired.

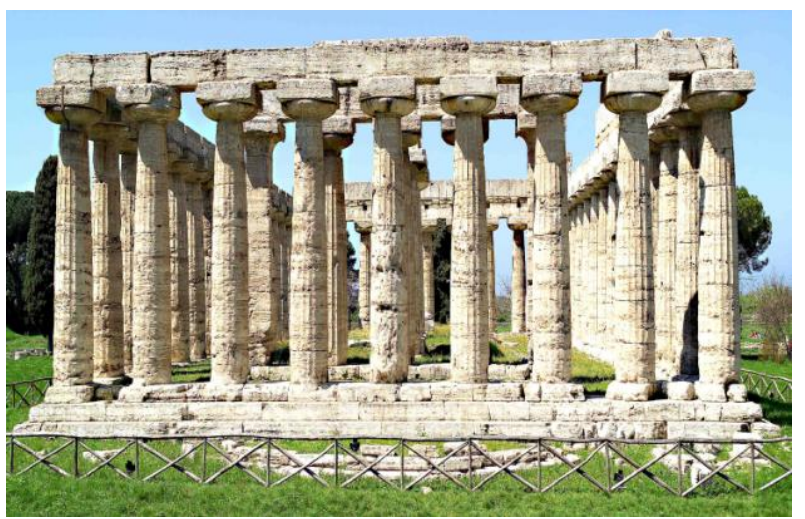

Figure 8. Hera Argiva's Temple in Paestum 
Photogrammetric shots relative to the Hera Argiva's Temple, also known as Basilica, have been taken by a calibrated Rollei camera characterized by a Phase One digital back, a wide - angle lens Zeiss optics with a focal length equal to 40 $\mathrm{mm}$, focal point $\mathrm{f}=4$; the photograms have been taken approximately at zenithal hour, similarly to what has been done for the Apollo's Temple in the archeological area of Pompei.

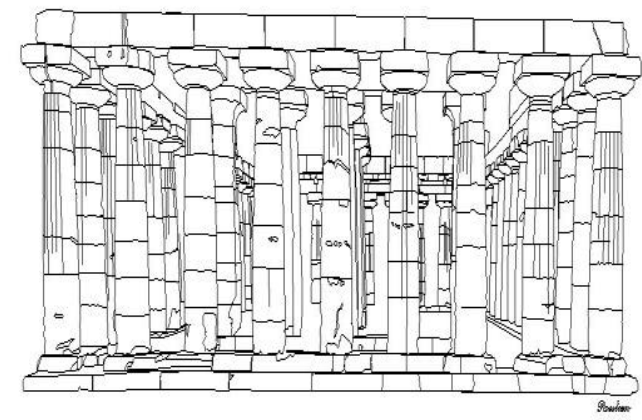

Figure 9. Axonometry Hera Argiva's Temple in Paestum

This is a necessary condition for the purpose of the survey, in order to properly catch the essentiality of the geometry of the monument since it is characterized by predominant vertical elements and stone material of light color. Also in this case output files have a dimension of more than 300 Megapixel and have allowed us a very accurate restitution. Moreover it has been possible to obtain a CAD image of the whole peristyle of all 50 column, allowing us a measurement of the volume and the load relative to the stone architrave, in travertine, as shown in Figure 9.

Hera Argiva'sTemple images, as it is apparent from the axonometry and by the detail illustrated in Figure 10, give evidence of a crack wide $14 \mathrm{~mm} \quad(0,1399 \mathrm{dm})$ approximatively; moreover, they allow for the control of the health status of stone materials with clear characterization of cracks, damages and travertine atomization. In particular the disgregation and atomization of stone material, known as "alveolization", for the presence of holes (alveolus), sometimes very deep, distributed in an irregular way on the surface of stone material, is consequence of the breaking up action exercised by the crystallization pressure of the salts into the pores.

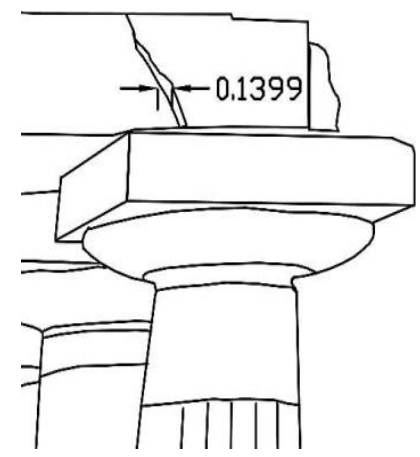

Figura 10. Particular of a fracture of Hera Argiva's Temple
The last study case, yet not less representative than the others, is the one pertaining to the Diver's Tomb, a structure of funeral art Magna Graecia, dated about 480 b.C. and represented in Figure 11. It is a case tomb, formed by 5 calcareous slabs in local travertine, which were accurately connected and filled at the time of their discovery.

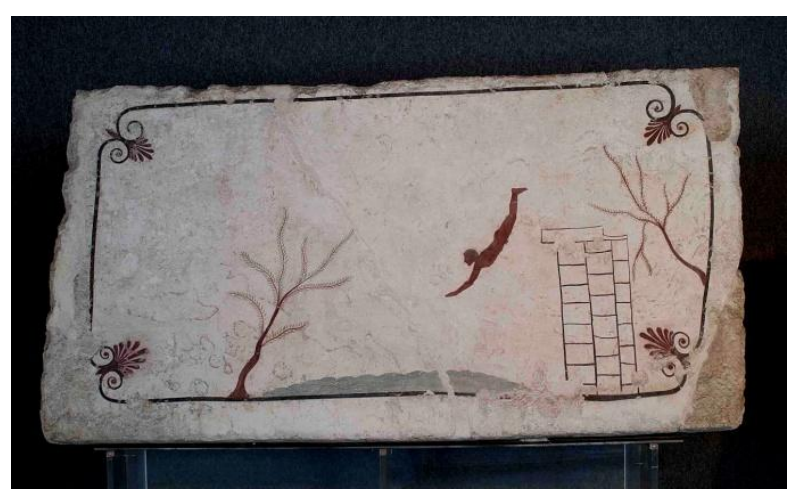

Figure 11. Diver's Tomb in Paestum

The image represents the metaphor of the passage from life to death and the instant when the young is dipping in water recalls the belief of old people that a river or a marsh might be passed through for reaching the world of deaths.

The exceptionality of this discovery, dated 1968 , is that the walls of the structures and the slab covering, appeared completely plastered and decorated with parietal paintings of figurative subjects.

To date this pattern, carried out with the fresco technique, is the only example of Greek painting relative to the classic age of Magna Graecia, a celebrated expression of Greek art, unfortunately almost fully lost. The photogrammetric shots relative to Diver's Tomb have been acquired with the calibrated metric Rollei camera and with a macro-planar Zeiss optics; moreover, the color temperature of the scene represented in the fresco has been measured by a Sekonic colorimeter.

The knowledge of the color temperature is mandatory for the restitution of the right coloring essential to the reproduction of the same color; in fact the restitution shown in Figure 12 has been subsequently "dressed" with colors as function of the on-site acquisition of the colors as is evident in Figure 13.
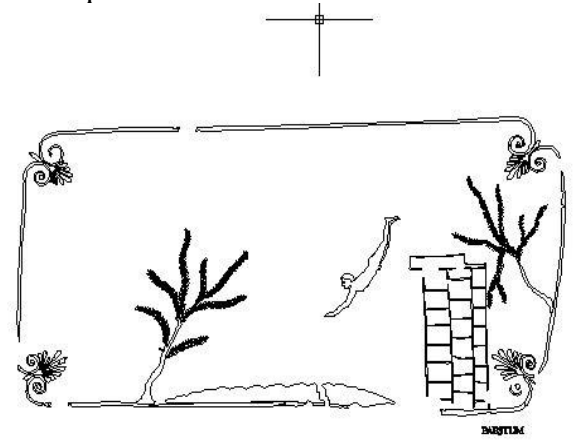

Figure 12. Restitution of Diver's Tomb

Thanks to these new advanced technologies a manual 1:1 colour reproduction of this wonderful exemplary, has been realized in ceramics material. 


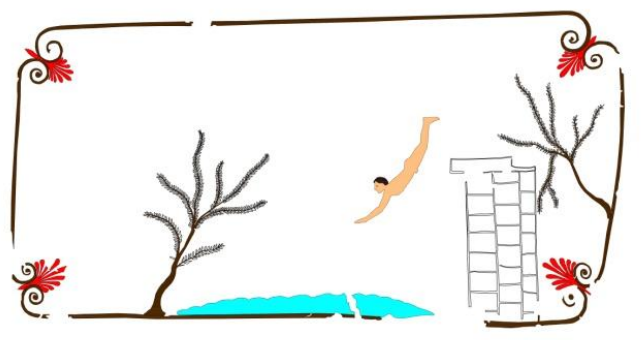

Figure 13. Color restitution of Diver's Tomb

Another slab of the sarcophagus, placed on the west side, represents a different scene with a procession open by a flute - player, followed by a man and a bearded and mantled old man, which leans himself on a stick. In the following figures the restitution and the color reproduction of this scene are shown.

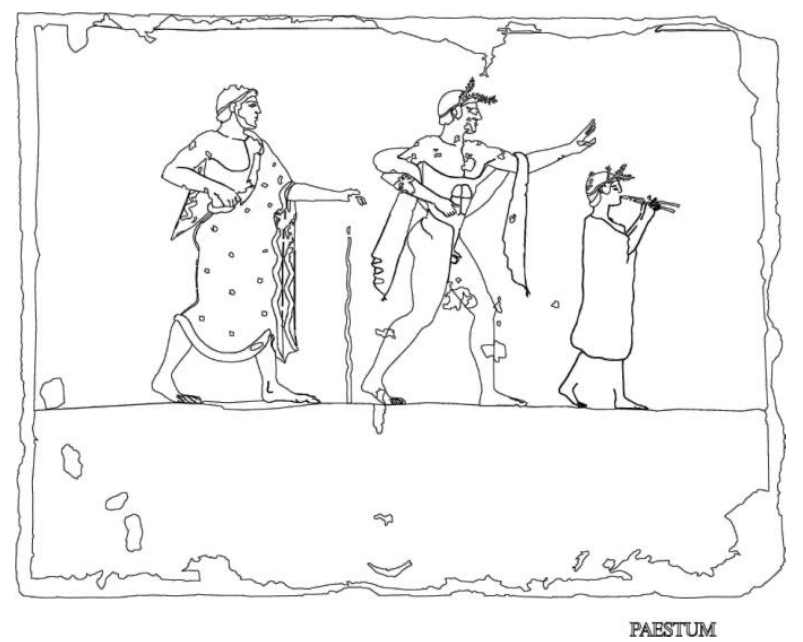

Figure 14. Restitution scene of the procession of Diver's Tomb

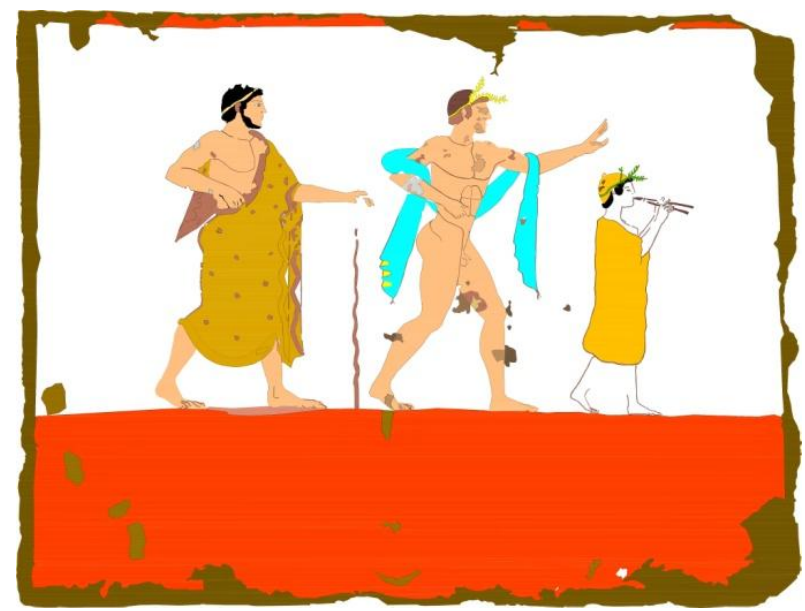

Figure 15. Color restitution scene of the procession of Diver's Tomb

\section{CONCLUSIONS}

The originality of this paper concerns an innovative approach to photogrammetric survey which combines the enhanced features of the informatical technologies with the accuracy of the traditional photogrammetric technique. In this sense the authors want to acknowledge Land's original experience, ingenious inventor of photography with instantaneous Polaroid finishing, who proved that the final perception of the color, whatever is point of the scene, depends by other colors perceived in other parts of the same scene. For this reason the authors have adopted a noncanonical approach to on-site acquisition. In fact the photogrammetric shots have been taken in conditions of brightness suited to the aim of the survey concerning, on one side, a geometry with prevalence of vertical lines, and on the other one, stone material with light color. Therefore, using specific measurement systems, the shots have been made with light value (length wave and color temperature known "a priori" and very high values of the parameter "exposure value "EV) compatible to an interpretation of the structures in opposition to a regular shot which, vice versa, wouldn't have considered the operative and environmental conditions as adequate. Moreover the difference between the results of our experience and the "rendering" relative to the same sites, is represented by the limited capability of the latter to raw algorithmic transformations. In fact the complete chromatic lack in the rendering doesn't allow for their use in applications, e.g., to show cracks and fractures, decay phenomena, as atomization of stone materials, moisture tracks, molds presence, etc. In addition the results obtained by this experience are well suited to whatever kind of subsequent digital video elaboration, suitable for a userfriendly diffusion and knowledge of archaeological and artistic heritage, particularly useful from a commercial point of view. Actually, in this respect, the final images are completely downloadable from web-sites, with the possibility to obtain very high printing quality and to know all data relative to each phase of elaboration process. In conclusion the classification of the huge Italian artistic and cultural heritage can find a proper framework within this study. Furthermore, the present contribution to the diffusion of the knowledge, the preservation and the protection of a rich historical-cultural heritage, often not enough known, becomes a part of a wider action aiming at improving the promotion of regional territory as well as the politics of incentives to cultural tourism's strategies, challenging the environmental damage, the territorial vulnerability and the safety.

\section{REFERENCES}

Ali M Reza, 2004 Realization of the contrast limited adaptive histogram equalization for real time image enhancement, Journal of VLSI Signal Processing, 38:35-44 Land E., 1959 Color vision and the natural image, part i Proceedings of the National Academy of Sciences of the United States of America, 45 (1):115-129; part ii 45 (4):636644

Napoli M., 1970 Il Museo di Paestum Editore Di MauroRichard E., Woods Rafael C.Gonzales , 2002 Digital Image processing, Prentice Hall, second edition

Zuiderveld K., 1994 in P.S. Heckbert editor, Graphic Gems, volume IV, chapter VIII.5, Academic Press, Cambridge, MA

Annex CIPA (CIPA DC-007-Translation-2009) Published by Camera \& Imaging Products Association JCII BLDG 25, Ichiban-cho, Chiyoda-ku, Japan 